\title{
DESIGN OF IIR EIGENFILTERS IN THE FREQUENCY DOMAIN
}

\author{
F. Argenti, E. Del Re \\ Dipartimento di Ingegneria Elettronica, Università di Firenze \\ Via di Santa Marta, 3 - 50139 Firenze - Italy \\ Tel.: +39554796424-Fax: +39554796485 \\ e-mail: argenti@cosimo.die.unifi.it
}

\begin{abstract}
In this work a method to apply the eigenfilter approach to the design of IIR filters in the frequency domain is described. 'The eigenfilter approach is an appealing way of designing digital filters, due mainly to the simplicity of its implementation: an error function is expressed as a quadratic form in the filter coefficients and the search of the eigenvector corresponding to the minimum eigenvalue yields the optimum solution. This technique has been successfully applied to the design of both FIR and IIR filters. In this work the IIR case is addressed: in our approach the error between a desired function and the response of a rational transfer function is expressed in the frequency domain. Some examples of numerical design are shown for different types of filters, lowpass and bandpass, to show the effectiveness of the presented method.
\end{abstract}

\section{INTRODUCTION}

the eigenfilter approach is a simple and flexible way of designing digital filters. The method consists in expressing the error between a target and a digital filter frequency response as a real, symmetric, positive-definite quadratic form in the filter coefficients. The error can be referred either to the time or to the frequency domain, or to both of them. The eigenvector corresponding to the minimum eigenvalue yields the optimum filter coefficients according to the chosen error measure

The method has been introduced for least-squares design of a variety of linear phase FlR digital filters in [1]. It has been extended to the case of FIR Hilbert transformers and digital differentiators in [2][3]. In [4] the eigenfilter approach has been applied to the design of FIR filters with an arbitrary frequency response and not having, in general, a linear phase.

Design of IIR eigenfilters in the time domain has been addressed in [5]. A clesired symmetrical infinite impulse response is considered as the sum of a causal and an anti-causal part. A recursive difference equation is used to approximate the causal part. 'The error between the target function and the impulse response of the system is expressed as a quadratic form in the filter coefficients, so that the eigenfilter approach can be applied. In this case, however. a noncausal system is necessary to implement the global desired impulse response. If a causal implementation is required, this can be obtained by substituting poles ontside the unit circle with their inverse conjugate and with a proper scaling of the transfer function: therefore, stable poles are constrained to be double. Moreover, the error weighting function operates in the time domain, making more complex a different consideration of the passbands and of the stopbands.

In [6][7] the eigenfilter approach is applied to the design of allpass sections with a given phase response. If two allpass sections are designed to be in-phase and out-of-phase in the passband and in the stopband, respectively, then the method can be applied also to the design of IIR filters [7][8]; however. the degrees of the numerator and of the denominator are related to the degrees of the allpass functions composing the system and can not be completely arbitrary.

Design of equiripple IIR filters with an arbitrary number of poles and zeros is dealt with, for example, in [9][10]. In [10] the solution of an eigenvalue problem yields the filter coefficients, even though the classical eigenfilter approach, based on the Rayleigh's principle [1] and on the search of the minimum eigenvalue of a positive-definite matrix, is not used.

In this work a new and simple method based on the eigenfilter approach to design causal IIR filters with an arbitrary number of zeros and poles is presented. It works in the frequency domain so that different weights can be assigned to the different bands. Moreover, no constraints are posed on zeros and poles of the system, so yielding more degrees of freedom.

The method is presented in the next section, while in Section 3 some examples of numerical design of IIR filters are shown.

\section{DESIGN OF IIR EIGENFILTERS}

The main problem in designing IIR eigenfilters is expressing the error between the target and the filter frequency response as a quadratic form in the filter coefficients. This task is easier in the FIR case, where the target function is not rational.

Let $H(z)$ be a rational function having $M$ zeros and $N$ poles (with arbitrary $M$ and $N$ ), i.e.,

$$
H(z)=\frac{b_{0}+b_{1} z^{-1}+\ldots+b_{M} z^{-M}}{a_{0}+a_{1} z^{-1}+\ldots+a_{N} z^{-N}}
$$

where $a_{n}, n=0,1, \ldots, N$, and $b_{n}, n=0,1, \ldots, M$ are real coefficients. Let $H^{d}(\omega)$ be a desired target function representing, in general, a lowpass, highpass, bandpass filter frequency response. Let $\omega_{k}$ be 
the bounds of each band (passband, transition band, stopband). For a lowpass filter, for example $\left(0, \omega_{1}\right)$, $\left(\omega_{1}, \omega_{2}\right)$ and $\left(\omega_{2}, \pi\right)$ are a passband, transition band and stopband, respectively,

Consider we are interested only in approximating the magnitude of the target function $H^{t t}(\omega)$. In our approach, however, we will assign also a phase to the function $H^{d}(\omega)$ : the initial guess will be a linear phase function. Therefore, if $H_{k}^{d}(\omega)$ is the target frequency response in the band $\left(\omega_{k}, \omega_{k+1}\right)$, we will assume:

a) $H_{k}^{d}(\omega)=e^{-i k w}$ if $\left(\omega_{k}, \omega_{k+1}\right)$ is a passband

b) $H_{k}^{d}(\omega)=f_{k}(\omega) e^{-j K \omega}$ if $\left(\omega_{k}, \omega_{k+1}\right)$ is a transition band

c) $H_{k}^{d}(\omega)=0$ if $\left(\omega_{k}, \omega_{k+1}\right)$ is a stopband

The choice of $K$ is discussed later. The real function $f_{k}(\omega)$ must be chosen so that it joins the amplitudes of the adjacent passband and stopband, i.e., it is a decreasing function with $f\left(\omega_{k}\right)=1$ and $f\left(\omega_{k+1}\right)=0$, or, viceversa, an increasing function with $f\left(\omega_{k}\right)=0$ and $f\left(\omega_{k+1}\right)=1$. In our examples, however, we will discuss mainly the case of transition bands considered as "don't care" bands.

In the ideal case, the frequency response $H(\omega)$ and $H^{d}(\omega)$ coincide, that is

$$
H^{d}(\omega)=\frac{b_{0}+b_{1} e^{-j \omega}+\ldots+b_{M} e^{-j M \omega}}{a_{0}+a_{1} e^{-j \omega}+\ldots+a_{N} e^{-j N \omega}}
$$

and, therefore, we should have

$$
\begin{gathered}
H^{d}(\omega)\left(a_{0}+a_{1} \epsilon^{-j \omega}+\ldots+a_{N} e^{-j N \omega}\right)= \\
=b_{0}+b_{1} e^{-j \omega}+\ldots+b_{M} \epsilon^{-j H \omega}
\end{gathered}
$$

However, since the above equation can not be verified, in general, for all the frequencies, an error function in the frequency domain can be defined for each interval $\left(\omega_{k}, \omega_{k+1}\right)$ :

$$
\begin{aligned}
E_{k}(\omega)= & H_{k}^{d}(\omega)\left(a_{0}+a_{1} e^{-j \omega}+\ldots+a_{N} e^{-j N \omega}\right)- \\
& -b_{0}-b_{1} e^{-j \omega}-\ldots-b_{M} e^{-j M \omega}
\end{aligned}
$$

A global error function $\Phi$ is given by:

$$
\Phi=\sum_{k} \beta_{k} \phi_{k}
$$

where $\beta_{k}$ is a constant that weighs the $k$-th band error function $\phi_{k}$ given by:

$$
\begin{aligned}
\phi_{k}= & \int_{-\omega_{k+1}}^{-\omega_{k}}\left|E_{k}(\omega)\right|^{2} W_{k}(\omega) d \omega+ \\
& +\int_{\omega_{k}}^{\omega_{k+1}}\left|E_{k}^{\prime}(\omega)\right|^{2} W_{k}(\omega) d \omega
\end{aligned}
$$

with $W_{k}(\omega)$ a positive weighting function.

If we consider the definitions of the following vectors

$$
\begin{aligned}
\mathrm{C}_{k}(\omega)= & {\left[H_{k}^{d}(\omega) H_{k}^{d}(\omega) e^{-j \omega} \ldots H_{k}^{d}(\omega) e^{-j N \omega}\right.} \\
& \left.-1-\epsilon^{-\omega} \ldots-\epsilon^{-M \omega}\right]^{T}
\end{aligned}
$$

$$
\mathbf{A}=\left[\begin{array}{llllllll}
a_{0} & a_{1} & \ldots & a_{N} & b_{0} & b_{1} & \ldots & b_{M}
\end{array}\right]^{T}
$$

then the error functions $\phi_{k}$ can be expressed as:

$$
\begin{aligned}
\phi_{k}= & \int_{-\omega_{k+1}}^{-\omega_{k}} \mathbf{A}^{T} \mathbf{C}_{k}^{\times}(\omega) \mathbf{C}_{k}^{T}(\omega) \mathbf{A} W_{k}(\omega) d \omega+ \\
& +\int_{\omega_{k}}^{\omega_{k+1}} \mathbf{A}^{T} \mathbf{C}_{k}^{*}(\omega) \mathbf{C}_{k}^{T}(\omega) \mathbf{A} W_{k}(\omega) d \omega
\end{aligned}
$$

where superscript $\mathrm{T}$ and $*$ denote transposition and conjugation, respectively $\left(\mathbf{A}^{*}=\mathbf{A}\right.$ since the filter coefficients are assumed real). Therefore, $\phi_{k}$ is given by:

$$
\phi_{k}=\mathrm{A}^{T} \mathrm{P}_{k} \mathrm{~A}
$$

where

$$
\begin{aligned}
\mathbf{P}_{k}= & \int_{-\omega_{k+1}}^{-\omega_{k}} \mathbf{C}_{k}^{*}(\omega) \mathbf{C}_{k}^{T^{\prime}}(\omega) W_{k}(\omega) d \omega+ \\
& +\int_{\omega_{k}}^{\omega_{k+1}} \mathbf{C}_{k}^{*}(\omega) \mathbf{C}_{k}^{T^{\prime}}(\omega) W_{k}(\omega) d \omega
\end{aligned}
$$

is a symmetric, real, positive-definite $(M+N+2) \times$ $(M+N+2)$ matrix. Therefore, the global error function $\Phi$ can be expressed as:

$$
\Phi=\mathbf{A}^{T}\left(\sum_{k} \beta_{k} \mathbf{P}_{k}\right) \mathbf{A}
$$

By using the eigenfilter approach, the optimal filter coefficients that minimize the error function $\Phi$ are the elements of the eigenvector of the matrix

$$
\mathbf{P}=\sum_{k} \beta_{k} \mathbf{P}_{k}
$$

corresponding to the minimum eigenvalue. 'The computation of the matrices $\mathbf{P}_{k}$ can be performed numerically in each band $\left(\omega_{k}, \omega_{k+1}\right)$.

Remarks:

1) Choice of the weight function $W_{k}(\omega)$ : As can be seen from the above discussion, we have to introduce a phase for the target function to express the error as a quadratic form in the filter coefficientis. However, we are interested only in approximating the magnitude of the target function: if the error $E_{k}^{\prime}(\omega)$ is small, then the magnitude constraint is approximately verified. In some previous works [1][4][7] a recursive updating of the weighting function was introduced to obtain an almost equiripple solution. In our case, the use of this recursive updating is recommended for the method to converge to a solution satisfying the magnitucle constraints.

Let. $\mathrm{A}^{(n)}$ be the solution vector at the $n$-th iteration and let $H^{(n)}(\omega)$ be the corresponding frequency response. 'The magnitude error in the $k-t h$ band is given by:

$$
e_{k}^{(n)}(\omega)=|| H_{k}^{d}(\omega)|-| H_{k}^{(n)}(\omega)||
$$

The weighting function to be used in the $(n+1)$-th iteration will be:

$$
W_{k}^{(n+1)}(\omega)=W_{k}^{(n)}(\omega) \operatorname{env}\left(e_{k}^{(n)}(\omega)\right)
$$


where env $(g(x))$ is the envelope of the positive function $g(x)$ (we used a linear interpolation between the maxima of $g(x)$ ). By using this approach, larger weights are assigned to larger errors until the weight function does not change any more, so that an almost equiripple solution is obtained at the end [1][4][7].

s) Iarget function phase updating: As discussed above, we try to approximate a linear phase function with a rational transfer function. Exact linear phase is obtained if poles appear in pairs, one the inverse of the other. By assuming a linear phase target function at each iteration, we found that one pole is usually placed in the transition band outside the unit circle. A causal stable system having the same magnitude of the designed frequency response can be obtained by substituting; at the denominator, the factor $(z-p)$ (where $p$ is the pole outside the unit circle) with $p^{*}\left(z-1 / p^{*}\right)$. As a drawback, a narrower: than required transition band can be obtained and this is often accomplished by assuming the transition bands not to be "don't care" bands: in other words, the magnitude and the width of the transition bands can not be easily controlled. Moreover, the number of poles that efficiently contribute to the response in the passband is reduced.

An improvement of the method consists in assuming as phase of the target function $H^{a}(\omega)$ at the $(n+1)$-th iteration, the phase of $H^{(n)}(\omega)$. Therefore, the method to design IIR eigenfilters can be summarized as follows:

Step 1. The pliase of the target function is initialized to be linear in the passbands and in the transition bands: we used $K$ of the order of $M / 2$.

Step 2. 'l'he matrix $\mathbf{P}$ is computed and the eigenvector corresponding to the minimum eigenvalue is found. An efficient method to accomplish this task is shown in [1].

Step 3. If unstable poles are found, they are substituted by their inverse conjugate and the frequency response is properly scaled.

Step 4. The target function $H^{d(n+1)}(\omega)$ to be matched at the $(n+1)$-th iteration is given by:

a) $H_{k}^{d(n+1)}(\omega)=e^{j<H^{(n)}(\omega)}$ if $\left(\omega_{k}, \omega_{k+1}\right)$ is a passband

b) $H_{k}^{d(n+1)}(\omega)=f_{k}(\omega) e^{j \angle H^{(n)}(\omega)}$ if $\left(\omega_{k}, \omega_{k+1}\right)$ is a transition band

c) $H_{k}^{d(n+1)}(\omega)=0$ if $\left(\omega_{k}, \omega_{k+1}\right)$ is a stopband

Step 5. The updating of the weight function $W_{k}^{(n)}(\omega)$ as shown in (15) is performed.

Step 6. Steps (2)-(5) are repeated until a stop criterion based on either filter coefficient or magnitude response variation is met.

In the next section some examples of numerical design are shown and discussed.

\section{EXPERIMENTAL RESULTS}

The effectiveness of the design method previously presented is shown through some numerical examples.
Example 1. Lowpass filter with the following characteristics: passband for $0 \leq|\omega| \leq 0.3 \pi$ and stopband for $0.4 \pi \leq|\omega| \leq \pi$. 'The orders of the numerator and of the denominator used to approximate this function are $M=9$ and $N=5$, while the weights $\beta_{0}=1$ and $\beta_{2}=2$ were chosen.

Example $2 . \quad$ Passband filter with the following characteristics: passband for $0.28 \pi \leq|\omega|<0.54 \pi$ and stopbands for $0 \leq|\omega| \leq 0.2 \pi$ and for $0.62 \pi \leq 1$ $\omega \mid \leq \pi$. This function is approximated by the frequency response of an IIR filter having $M=N=12$. The weights $\beta_{0}=\beta_{2}=\beta_{4}=1$ have been used.

In these examples we have considered the transition bands as "don't care" bands. The integrals that appear into the definition of the matrices $\mathbf{P}_{k}$ have been computed numerically by using a grid of 200 points in each band. The updating of the weighting function and the searching of a new set of coefficients stops when $\max \left(\left|\mathbf{A}^{(n+1)}-\mathbf{A}^{(n)}\right|\right)<10^{-3}$ or $\max _{k} \max _{\omega}\left(|| H_{k}^{(n+1)}(\omega)|-| H_{k}^{(n)}(\omega)||\right)<10^{-4}$.

The results relative to Example 1 and 2 obtained by using our design method are presented in Fig. 1 and 2, respectively; the frequency response of the filters and a zoom of the passband detail are shown. As can be seen, the frequency response of the designed filters is almost equiripple in each band.

\section{CONCLUSIONS}

In this work a method for applying the eigenfilter approach, based on the Rayleigh's principle, to the design of IIR digital filters is shown. One of the most appealing features of the eigenfilter approach is its simplicity, since the problem is reduced to the search of the minimum eigenvalue and of the corresponding eigenvector of a real, symmetric, positivedefinite matrix. 'The method includes also an iterative procedure to update the weighting function, so that an almost equiripple solution is obtained.

\section{REFERENCES}

[1] P.P. Vaidyanathan, 'L'.Q. Nguyen, "Ëigenfilters: A New Approach to Least-Squares FIR Filter Design and Applications Including Nyquist Filters", IEEE' I'rans. Cincuits Syst, Vol. CAS-34, no. 1, Jan. 1987, pp. 11-23.

[2] S.C. Pei, J.J. Shyu, "Design of FlR Hilbert Transformers and Differentiators by Eigenfilter", IEEE Trans. Circuits Syst, Vol. 35, no. 11, Nov. 1988, pp. 1457-1461.

[3] S.C. Pei, J.J. Shyu, "Eigenfilter Design of Higher-Order Digital Differentiators", IEt' $E^{\prime}$ Irans. Signal Processing, Vol. 37, no. 4, Apr. 1989, pp. 505-511.

[4] 'I.Q. Nguyen, "The Design of Arbitrary Fll Digital Filters Using the Eigenfilter Method". IEEE Trans. Signal Processing. Vol. 41, no. 3 , Nov. 1993, pp. 1128-1139.

[5] S.C. Pei, J.J. Shyu, "Design of 1-D and 2-D IIR Eigenfilters", IELE Trans. Stgnal Processing, Vol. 42, no. 4, Apr. 1994, pp. 962-966. 
[6] S.C. Pei, J.J. Shyu, "Eigenfilter Design of 1$D$ and 2-D IIR Digital All-Pass Hilters", IEEE Irans. Signal Processing, Vol. 42, no. 4. Apr. 1994, pp. 966-968.

[7] 'T.Q. Nguyen: T.1. Laakso, R.D. Koilpillai, "Eigenfilter Approach for the Design of Allpass Filters Approximating a Given Phase Response", IEEE Trans. Signal Processing, Vol. 42, no. 9, Sep. 1994, pp. 2257-2263.

[8] S.C. Pei, J.J. Shyu, "Design of IIR multiband filters using IIR all-pass filters", Signal Processing, Vol. 37, 1994, pp. 87-94.

[9] L.B. Jackson, "An Improved Martinez/Parks Algorithm for IIR Design with Unequal Numbers of Poles and Zeros", IEEE Trans. Signal Processing, Vol. 42, no. 5, May 1994, pp. 12341238.

[10] X. Zhang; H. Iwakura "Desing of IIR Digital Filters Based on Eigienvalue Problem", IEEE Trans. Signal Processing, Vol. 44, no. 6, June 1996, pp. 1325-1333.

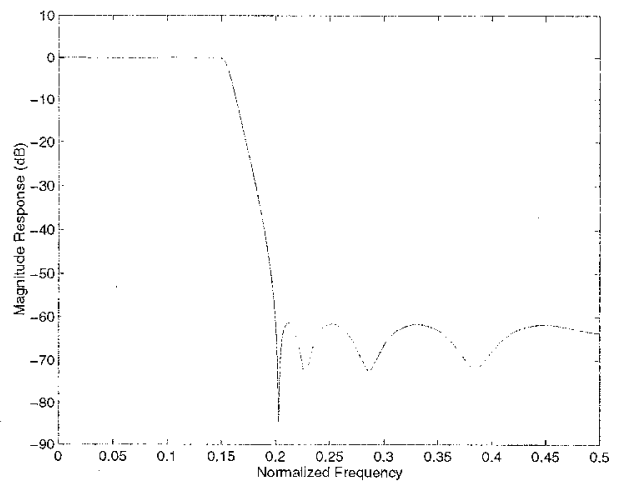

(a)

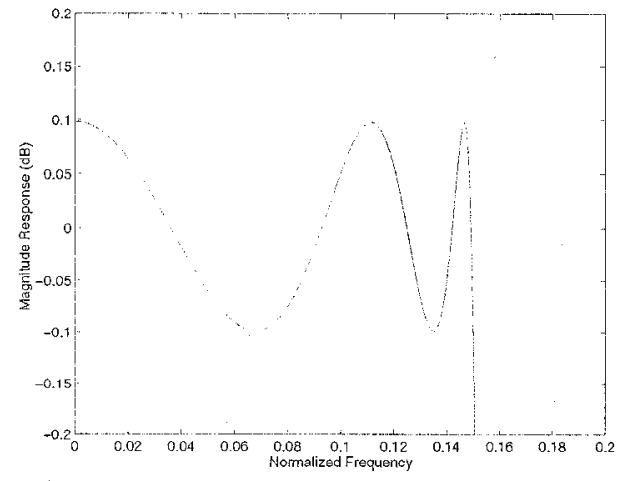

(b)

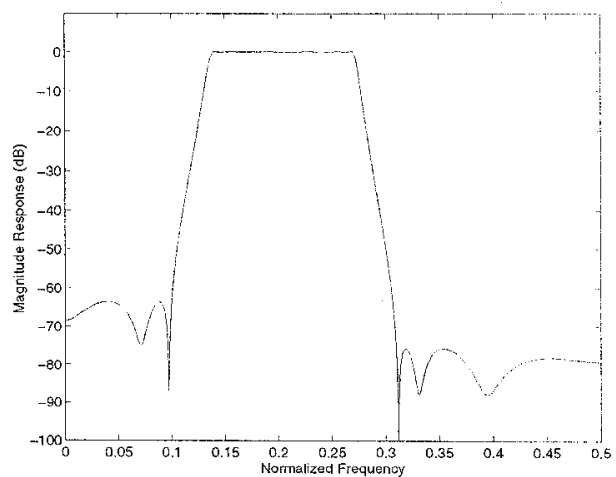

(a)

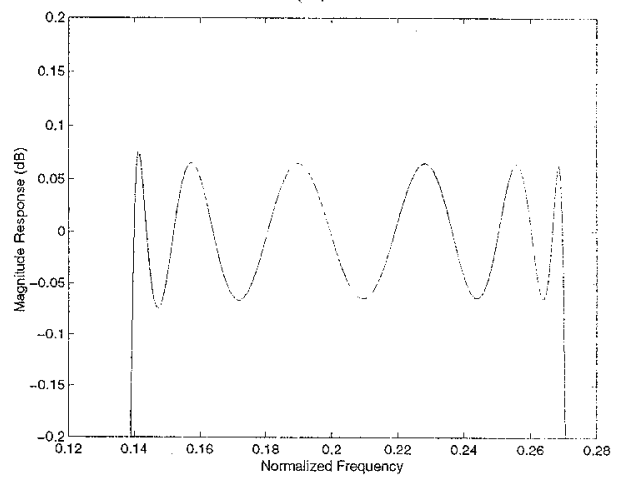

(b)

Fig. 2. Example 2: (a) Frequency response obtained with $M=N=12 ;$ (b) passband detail.

Fig. 1. Example 1: (a) Erequency response obtained with $M=9$ and $N=5 ;(b)$ passband detail. 